\title{
Object-Oriented Dynamic Modelling of Gas Turbines for CSP Hybridisation
}

\author{
Luis J. Yebra ${ }^{1,7}$ Sebastián Dormido $^{2} \quad$ Luis E. Díez $^{3} \quad$ Alberto R. Rocha ${ }^{4} \quad$ Lucía González $^{4}$ \\ Eduardo Cerrajero $^{5}$ Silvia Padilla ${ }^{6}$ \\ ${ }^{1}$ Plataforma Solar de Almería, CIEMAT, Spain, luis . yebra@psa.es \\ ${ }^{2}$ Dpto. Informática y Automática, UNED, Spain, sdormido@dia. uned.es \\ ${ }^{3}$ SERLED Consultores, Spain, le.diez@serled.com \\ ${ }^{4}$ ACS-Cobra T\&I, Spain, \{arocha@acsindustria.com, luciagonzalez@grupocobra.com\} \\ ${ }^{5}$ IDIE (Investigación, Desarrollo e Innovación Energética), Spain, eduardo. cerra jero@idie.es \\ ${ }^{6}$ AITESA (Air Industrie Thermique), Spain, spadillaeaitesa.es \\ ${ }^{7}$ CIESOL, Joint Centre of the University of Almería-CIEMAT, Spain
}

\begin{abstract}
This paper presents a dynamic model of a gas turbine developed for the HYSOL project. The model is developed mainly for control purposes and based on mathematical, physical and chemical principles. Approximations and assumptions are presented with the objective to minimize complexity and to maintain a modular structure. The main modules are presented independently and ready to be connected to form the complete and parameterizable gas turbine model. Possible cases of algebraic loops appearance are detected and solutions are proposed to avoid them. Moreover, first principles compression and expansion maps are developed to avoid non-linear algebraic loops. The Modelica modelling language and the libraries Modelica.Fluid and Modelica.Media have been extensively used for the models development. Results from simulation experiments are presented, implementing the proposed mathematical models for compressor and turbine submodules independently, as well as for the complete gas turbine system.

Keywords: CSP hybridisation, gas turbine, object oriented modelling, first principle compression/expansion maps, simulation of gas turbines
\end{abstract}

\section{Introduction}

Mathematical approximations applied in the dynamic modelling of a gas turbine for model based control purposes are presented in this paper. The hypothesis presented come from the first principles used in a wide range operation model to be used in HYSOL european project. The information presented in this paper comes exclusively from published sources and detailed through references mainly focused in the technology behind the Modelica modelling language. Suggested reads for the Modelica language are (Cellier, 1991; Åström et al., 1998; Fritzson, 2004). For turbines concepts it has been mainly used (Bathie, 1996; Kehlhofer et al., 2009; Gülen and Kim, 2014). An important reference of previous imple- mentations concepts is the ThemoPower Modelica library (Casella and Leva, 2006).

In this paper, the modelling principles for the three main gas turbine subsystems are presented, oriented to be implemented in the Modelica modelling language. Modelica is a general purpose acausal object-oriented modelling language for physical systems modelling (Fritzson, 2004),

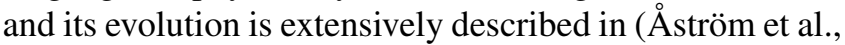
1998). The Modelica Standard Library (MSL) is frequently referenced for base classes and final use models to be directly reused in gas turbine subsystems components. Modelica.Media and Modelica.Fluid are the two most used packages from MSL in the presented work. For modelling and simulation works the Dymola(c) tool (3DS, 2016) has been used.

\section{Description of a Basic Gas Turbine Model}

This section presents the approximations used in the modelling the different parts, all obtained from public references. The two main phenomena background come from thermofluid and mechanical disciplines. Object oriented thermofluid modelling is described in (Tummescheit, 2002), which indeed has its base in the Computational Fluid Dynamics (CFD) methods detailed in (Patankar, 1980) and (Versteeg and Malalasekera, 1995). Currently the Modelica.Fluid library implement these concepts, see (Elmqvist et al., 2003). With respect to mechanical components it is only required to model 1-dimensional rotational mechanical systems and therefore the Modelica. Mechanics. Rotational package has been directly used.

Following the above references, the modelling methodology for thermofluid parts use two main concepts detailed in (Tummescheit, 2002):

- Control Volumes (CV). Stating mass and energy conservation, computing effort variables from flows. 


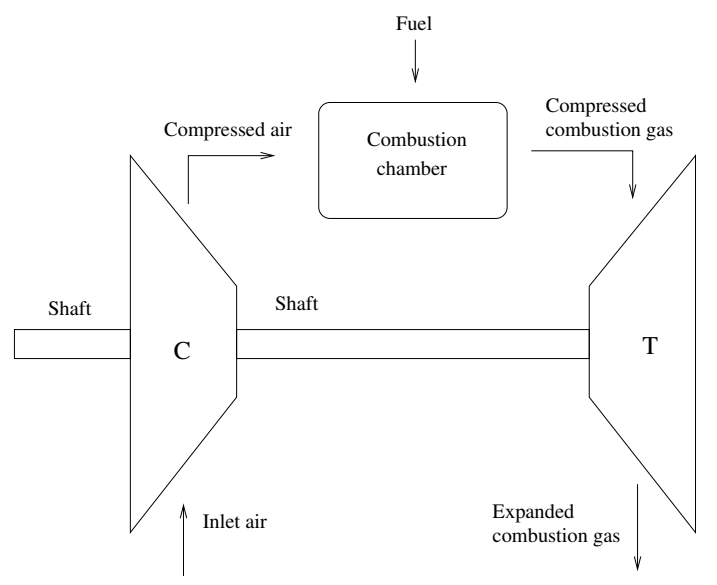

Figure 1. A general gas turbine diagram composed by compressor ('C'), combustion chamber, turbine ('T') and shaft. These are the minimum components to be considered for a basic turbine in an object oriented approach.

- Flow Models (FM). Stating momentum conservation, and computing flows from effort variables.

The general scheme regarded for the gas turbine is depicted in Fig. 1, referenced as basic gas turbine engine (Bathie, 1996), in which three main connected components are shown: compressor, combustion chamber and turbine. The compressor and turbine are connected mechanically by a shaft with other secondary components that are usually present in detailed models but not regarded in the analysis presented in this article.

For definition of effort and flow variables, see (Cellier, 1991). So all components will be classified as CV or FM, or an alternating arrangement of them: ...-CV-FMCV-FM...

So all the components in this model will be classified in either of both categories: CV or FM, attending to minimize the order of the final differential and algebraic equation (DAE) system, that is, trying to minimize the number of components of type CV.

As detailed in (Kehlhofer et al., 2009) and (Bathie, 1996), the boundary conditions defined by environment air pressure, temperature and humidity strongly influence the behaviour of the turbine-set. Hereafter turbine-set is referred as the set composed by compressor-combustion chamber-turbine, that is our basic gas turbine. Interesting qualitative analysis about changes in operational point of the turbine-set w.r.t. environment variables changes is presented in two chapters in (Kehlhofer et al., 2009). So, any model to be used in a wide range conditions should accept variations at inlet air conditions. These variations should be compensated by the control system. The Modelica. Media library (Casella et al., 2006) has been used to define the set of classes that implement different mediums properties.

The turbine-set model will be used in model based control applications that would require mathematical model inversion. This usage obligates to apply assumptions that minimize model complexity and let to obtain the required mathematical inverse model.

\subsection{Compressor}

There exist different types of compressors with a variety of complexities and applications: axial, rotary, centrifugal and reciprocating. The model presented in this section is general and simpler than (Greitzer, 1976), assuming that the compressor is an idealized FM device in which neither mass nor energy conservation are stated, and the isentropic conditions are met in the operating range. Extreme situations like surge and stall instabilities, and choked flow conditions, are never met. Ideal gas state assumption is used for the air.

Isentropic conditions assume that the state of the gas changes through the compressor under the condition $d s=$ 0 for any general formulation of balances of mass and energy. The general definition for specific entropy $s$ appears as its classical definition equation:

$$
T d s=d u+p d v
$$

where, in a differential mass element $d m, T$ is the temperature, $u$ is the specific internal energy, $p$ the pressure, $v=1 / \rho$ the specific volume and $\rho$ the density.

From the mechanical point of view, inside the compressor, there is an interaction between the gas flow (assumed isentropic flow) and the shaft blades that is stated in terms of a steady state energy balance as shown in (2):

$$
\dot{m} \Delta h=\tau \omega \eta_{\text {mec }}
$$

where $\dot{m}$ is mass flow rate, $\Delta h=h_{\text {outlet }}-h_{\text {inlet }}$ is the specific enthalpy increment through the compressor, $\tau$ is the torque applied over the shaft, $\omega$ the angular velocity of the shaft and $\eta_{m e c}$ the mechanic efficiency. The previous equation is actually an energy balance not usual in a FM, but necessary to link the thermodynamic and mechanical domains.

In practice, the parameters available from industrial compressors are usually implicit in the measured compressor characteristic (or map) from the company manufacturer. The compressor map is a 2-D table with its steady state operation points, that relates the pressure ratio $\Phi_{c}$ with the mass flow rate $\dot{m}$ and angular velocity $\omega$. From the map, the $\Phi_{c}(\dot{m}, \omega)$ could be fitted from a polynomial interpolation in these two supposed independent variables $\dot{m}$ and $\omega$. So the next equation could be assumed as constitutive for the component:

$$
\frac{p_{o}}{p_{i}}=\Phi_{c}(\dot{m}, \omega)
$$

where $p_{o}$ and $p_{i}$ are the output and input pressures of the compressor.

In the case of the computation of the specific enthalpy increment there are several approximations in the literature. Under isentropic conditions (4) is used: 


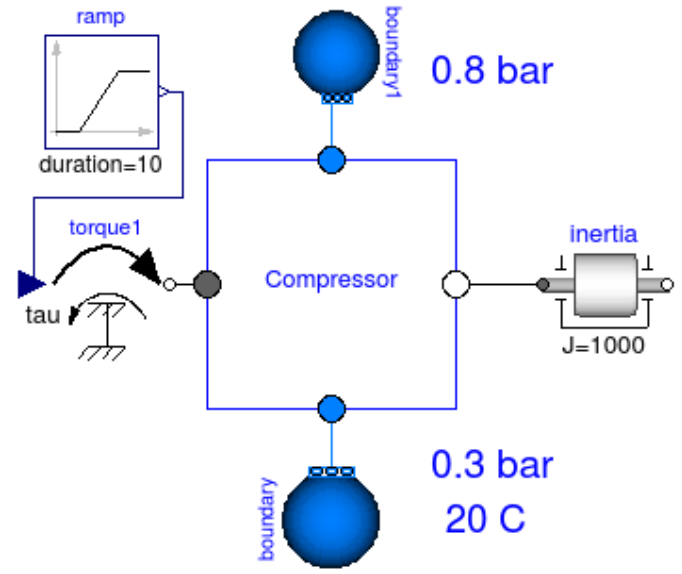

Figure 2. Diagram of the compressor in Dymola tool. The model implements the compressor map from one example of ThermoPower library.

$$
\Delta h \approx \Delta h_{s}=\left(\left(\frac{p_{o}}{p_{i}}\right)^{\frac{\kappa-1}{\kappa}}-1\right) c_{p} T_{i}
$$

where $\Delta h_{s}$ is the enthalpy increment under isentropic conditions, $\kappa$ the isentropic exponent, $c_{p}$ is the specific heat at constant pressure, and $T_{i}$ gas inlet temperature.

In those cases in which the approximation $\Delta h \approx \Delta h_{s}$ is not acceptable (5) could be applied, where $\eta$ is the isentropic efficiency.

$$
\eta(\dot{m}, \omega)=\frac{\Delta h_{s}}{\Delta h}
$$

Isentropic efficiency is a part of the compressor maps too, depends on $(\dot{m}, \omega)$, and can be considered as another constitutive equation for the component.

In general, compressor maps are formed by $\Phi_{c}(\dot{m}, \omega)$, $\eta(\dot{m}, \omega)$, among other data. These maps are obtained by experimentation in steady state conditions assuming that $\dot{m}$ and $\omega$ are independent variables in the test. This experimentation produces data sets that not always perform the best under dynamic simulations and transformation to other independent variables set is recommended. Transformation to beta lines independent variables is usually applied for turbine specific simulation software.

Fig. 2 presents a schematic diagram in Dymola showing the experiment simulated for the compressor. The compressor model is implemented using isentropic approximations and isentropic efficiency, using all components from Modelica.Fluid and Modelica.Media. The gas used as medium is dry air as ideal gas.

Fig. 3 shows the evolution of angular velocity of the shaft omega, and the mass flow rate mdot_compressor are the simulated variables, under the shown boundary conditions in which the driving torque Tu_ext is the input. The compressor is loaded by an inertia component from
Modelica.Mechanics.Rotational representing the shaft whose initial angular velocity is computed by Dymola. The torque positive ramp increments the angular velocity under constant thermofluid boundary conditions.

\subsection{Combustion Chamber}

This component performs the combustion of pressurized air with the fuel used. The usual fuel used in gas turbines are hydrocarbons, with a general chemical formula of $C_{x} H_{y}$. These reactions generate heat flow incoming from enthalpy of combustion.

Dynamic modelling of chemical reactions in general is clearly exposed in (Cellier, 1991), in the former modelling language for the Dymola tool. The dominant time constants of these kind of reactions are considerably smaller than the lowest one from thermofluid and mechanic dynamics. When the chemical reaction models are regarded, the DAE finally obtained for the complete gas turbine model become too stiff. The stiffness is a numerical property usually not welcome, but has to be accepted when it is needed to know internal information of the reaction evolution. Due to the main objective of this model is to predict thermal and mechanic dynamics, it was preferred to neglect the chemical dynamical behaviour, considering it as a non modelled dynamics. So, in this case, no composition changes are assumed in this model.

In this way, using Modelica components, the model used for the combustion chamber was a $\mathrm{CV}$ from Modelica.Fluid(.Vessels.ClosedVolume), in which a prescribed heat flow is injected, emulating the heat flow incoming from continuous hydrocarbon burning gas flow. The heat flow will be calibrated with experimental data from the facility, depending on the hydrocarbon composition and flow rate. Fig. 4 shows the composition diagram.

Further modelling work for this component will be reaction modelling for the prediction of the composition variation, when needed.

\subsection{Turbine Module}

There are two general types of turbines, the radial-flow and axial-flow turbines, as detailed in (Bathie, 1996). In industrial applications the turbine module is composed of several submodules connected. But beyond the technical and engineering details behind turbines, the interesting point is that it is a device with deep similarities in the physical behaviour with the compressor. Although in this case, the gas is expanded when passing through it making mechanical work on the shaft.

For the previous reasons the turbine module is considered a FM in thermofluid modeling methodology, although again, an internal steady state energy balance must be formulated to link thermofluid and mechanical domains. So, (2) applies for both: turbine and compressor.

In general, from first principles point of view, the mathematical model presented in section 2.1 is applied in the turbine module, with the same requirement that the tur- 

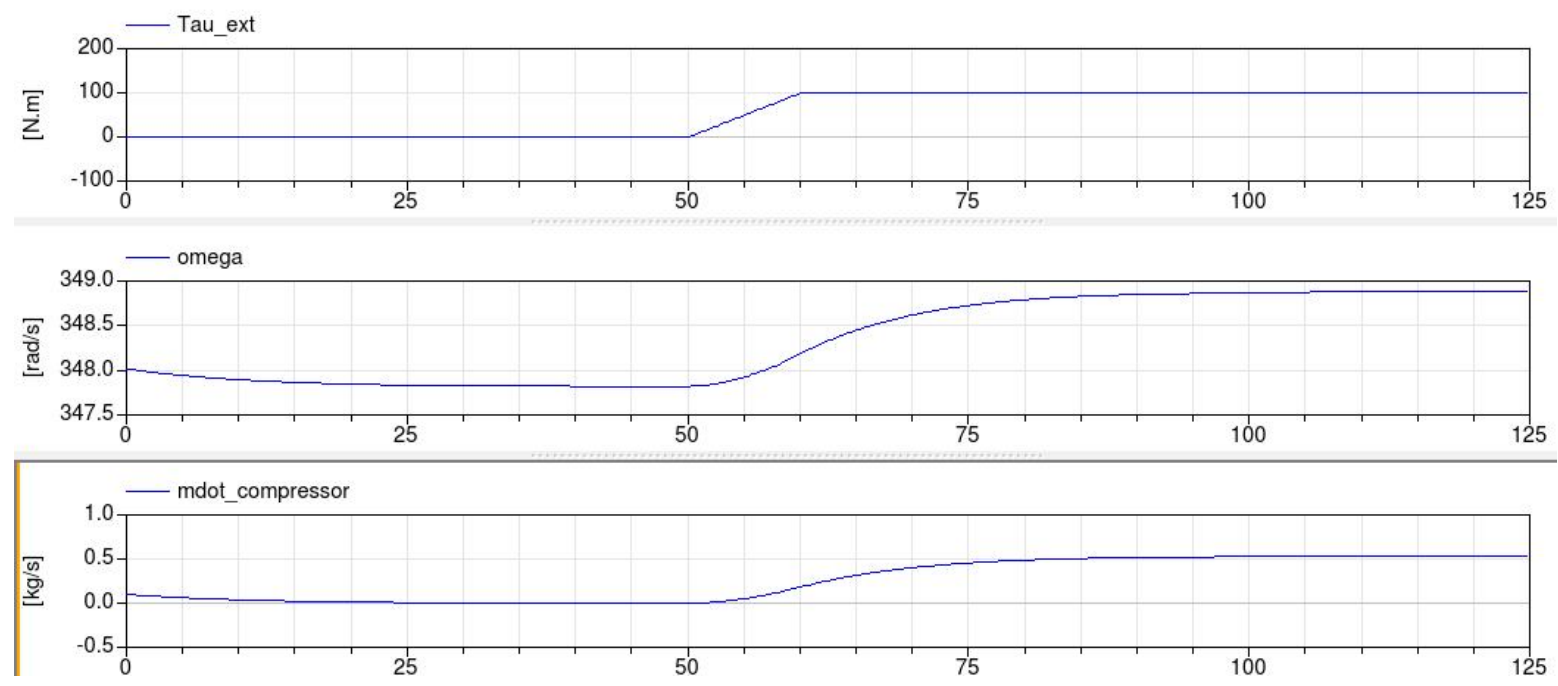

Figure 3. Simulation of the compressor under constant thermofluid boundary conditions and a ramp in the driving torque.

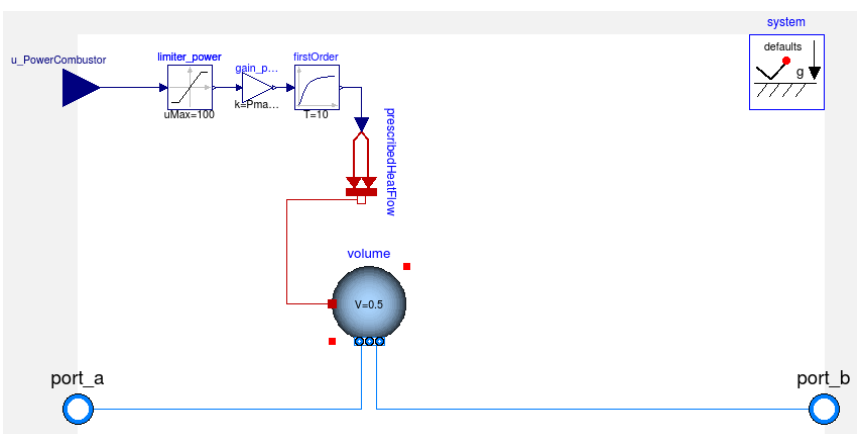

Figure 4. Diagram of the combustor in Dymola tool. The model implements a CV in which the air is heated by the combustion enthalpy, estimated outside the component.

bine maps $\left(\Phi_{c}(\dot{m}, \omega), \eta(\dot{m}, \omega)\right)$ must be provided. These maps are obtained usually from steady state measurements under certain conditions (although in the turbine case the word map is not used so frequently as in the compressor case). So, from an object oriented point of view, could be possible to define the physical model once for both, the compressor and turbine module, in a partial base class from which both could be inherited and parameterized by their compression/expansion maps $\left(\Phi_{c}(\dot{m}, \omega), \eta(\dot{m}, \omega)\right)$.

Fig. 5 shows a schematic diagram in Dymola showing the experiment simulated for the turbine module. The turbine module model implemented used isentropic approximations using all components from Modelica.Fluid and Modelica.Media. The gas used is dry air as ideal gas. Fig. 5 shows the inertia component representing the shaft inertia, over which a torque ramp is applied trying to simulate a disturbance.

Fig. 6 depicts the evolution of angular velocity of the shaft omega, and the mass flow rate mdot_compressor. Both are the simulated variables, under the unique boundary condition that is the driving torque Tu_ext. This torque emulates a brake acting on

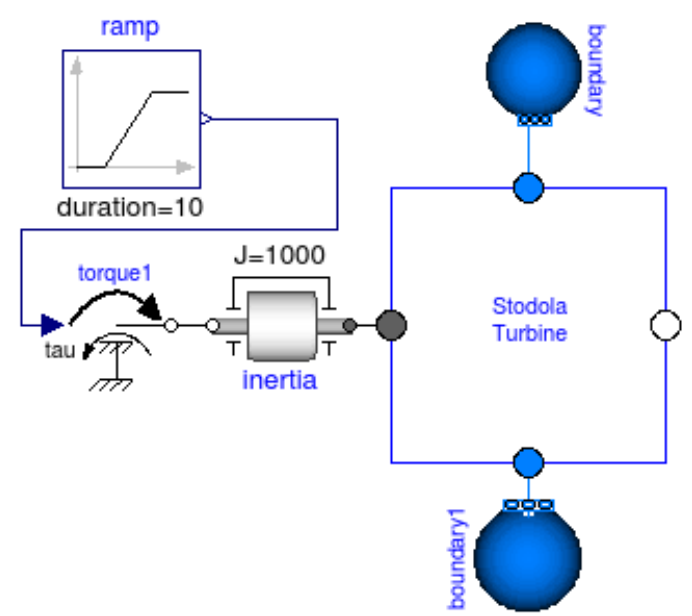

Figure 5. Diagram of the turbine in Dymola tool. The model implement the turbine map from one example of ThermoPower library.

the shaft. As in the compressor case, the initial value for the angular velocity of the shaft is computed by Dymola. The torque negative ramp decrements the angular velocity under constant thermofluid boundary conditions.

\section{Computational Causality And Con- ditions for Numerical Convergence}

Dymola tool will apply several symbolic manipulation algorithms to formulate the DAE of the turbine-set, and to define the set of algebraic equations to successfully find initial conditions. This section presents a simplified set of equations for the initialization of the DAE, formerly presented and ordered by hand trying to find the minimum number of algebraic loops. That is, computational causality has been calculated manually and the variables between brackets ([ ]) represent the computed variable from that equation. 

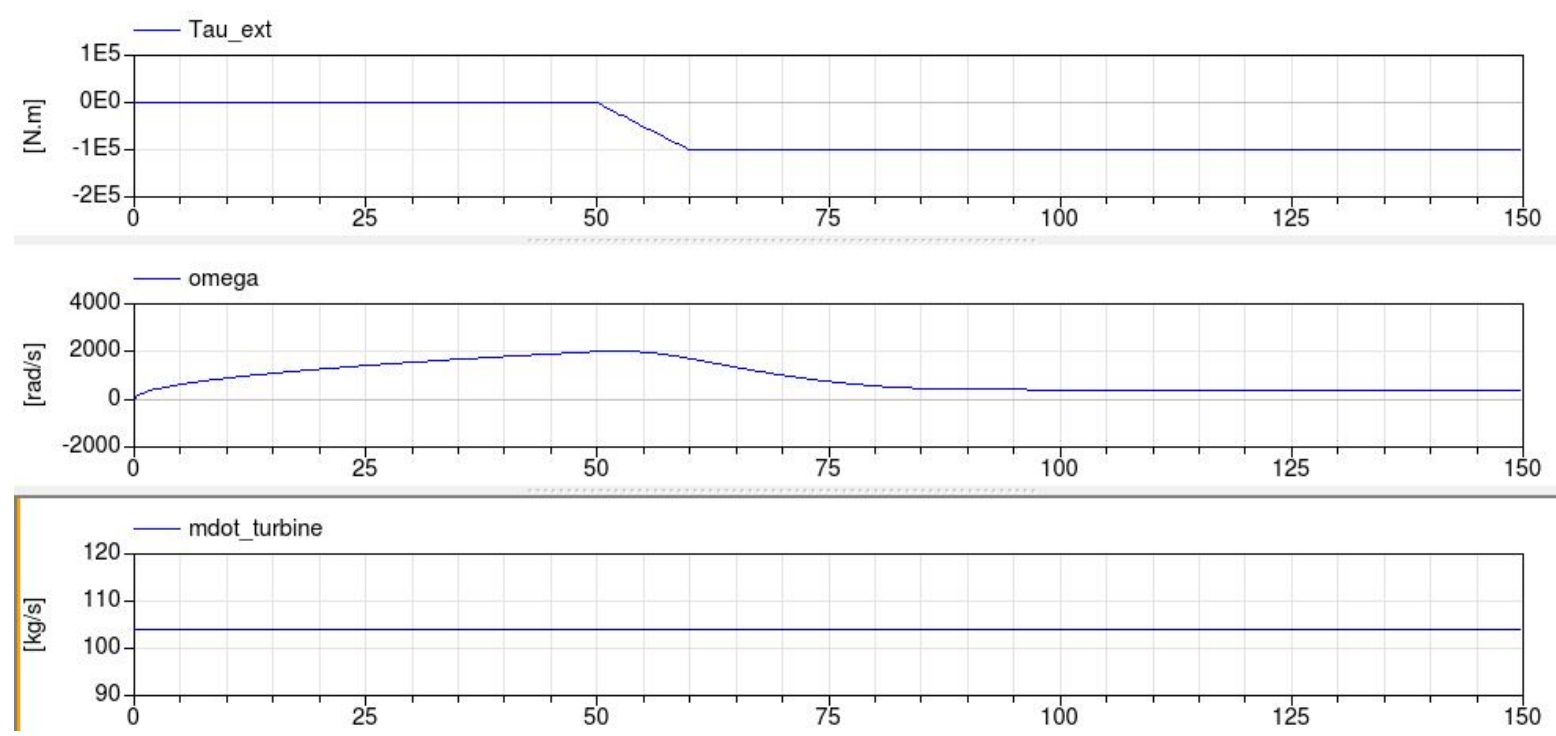

Figure 6. Simulation of the turbine under constant thermofluid boundary conditions and a ramp in the driving torque acting over the inertia.

The known variables are $\left\{\omega, p_{t, o}, p_{c, i}\right\}$ and the unknowns are $\left\{\dot{m}, \tau_{c}, \tau_{t}, \Delta h_{c}, \Delta h_{t}, \dot{\omega}\right\}$. Where subscripts 'c', 't', 'o', 'i' stand for: compressor, turbine, outlet and inlet, respectively. Parameters are: $\left\{\eta_{\text {mec }, c}, \eta_{\text {mec }, t}, J\right\}$. Properties: $\left\{\Phi_{c}, \eta_{c}, \Phi_{t}, \eta_{t}\right\}$.

The ordered list of equations:

$$
\begin{gathered}
\frac{p_{t, o}}{p_{c, i}}=\Phi_{c}([\dot{m}], \omega) \Phi_{t}([\dot{m}], \omega) \\
{\left[\Delta h_{c}\right]=\eta_{c}(\dot{m}, \omega)} \\
\dot{m} \Delta h_{c}=\left[\tau_{c}\right] \omega \eta_{m e c, c} \\
{\left[\Delta h_{t}\right]=\eta_{t}(\dot{m}, \omega)} \\
\dot{m} \Delta h_{t}=\left[\tau_{t}\right] \omega \eta_{m e c, t} \\
\tau_{c}+\tau_{t}=J[\dot{\omega}]
\end{gathered}
$$

Only in the first equation from the list, (6), an algebraic loop appears involving the compressor and turbine maps. Usually is a non-linear equation that must be solved by numerical methods, although it is important to note that the maps should be tested for numerical convergence of this equation.

Actually the choice of known variables is similar to that of the dynamic initial value problem (IVP) formulated, so the testing of convergence of (6) should be mandatory.

The model presented in section 2 based in Modelica.Fluid and Modelica.Media is more complex and the causality analysis performed by Dymola could vary, but the conclusion about the necessary good numerical behaviour of compression and expansion maps applies too, beside new others.

\section{First Principles Compression and Expansion Maps}

\subsection{Compression Case}

Compression maps are usually obtained from steady state measurements or data from manufacturer. When those maps are not available, first principle solutions may be implemented. In (Greitzer, 1976; Gravdahl and Egeland, 1997, 1999; Gravdahl et al., 2000, 2004) a description of the physical principles used in the deduction of a generic compression map are presented and more references for deeper details can be found. Based on these, may be concluded that $\Delta h_{s}$ in (4) could be approximated under some assumptions by (12).

$$
\Delta h_{s}=\sigma r_{2}^{2} \omega^{2}-\frac{r_{1}^{2}}{2}(\omega-\alpha \dot{m})^{2}-k_{f} \dot{m}^{2}
$$

Under isentropic conditions, the main contribution of this formulation is that $\Delta h_{s}$ is formulated as an algebraic relation with a second-degree polynomial in $\dot{m}$, that let us easily rearrange (12) to obtain an explicit relation for $\dot{m}$, obtaining (13). This choice avoids non linear algebraic loops in (6).

$$
\dot{m}=\frac{\alpha \omega r_{1}^{2} \pm \sqrt{D(b, \omega)}}{2 a_{2}}
$$

where:

$$
\begin{gathered}
D(b, \omega)=\alpha^{2} \omega^{2} r_{1}^{4}-4 a_{2}\left(a_{01} \omega^{2}+b\right) \\
b\left(\Phi_{c}, c_{p i}, T_{i}\right)=\left(\Phi_{c}^{\frac{\kappa-1}{\kappa}}-1\right) c_{p i} T_{i}
\end{gathered}
$$

In (12), (13) and (14) a new set of six parameters appears: $\left\{\sigma, \alpha, r_{1}, r_{2}, k_{f}, a_{01}, a_{2}\right\}$ where only the first 
five are independent, being $\left\{a_{01}, a_{2}\right\}$ dependent of them. Please, note that $b$ depends on the boundary conditions for the case of a compressor model. For more details, please read section 7 .

The conditions for model validity are defined in equations (16) and (17), and the model will be singular if unfulfilled any of both. This is convenient to consider in the initialization code.

$$
\begin{gathered}
\omega \geq \omega_{\min }(b)=2 \sqrt{\frac{a_{2} b}{\alpha^{2} r_{1}^{4}-4 a_{2} a_{01}}} \\
\dot{m} \geq \dot{m}_{\min }(b)=\frac{\alpha \omega_{\min } r_{1}^{2}}{2 a_{2}}
\end{gathered}
$$

\subsection{Expansion Case}

For the turbines, similar equations should be derived. A typical extended case is the Stodola turbine model, with results presented in section 2.3 , in a simplified way to avoid the general approach described in section 3 , expressed by (18) and (19).

$$
\begin{gathered}
\dot{m}=K \sqrt{\frac{p_{i}}{T_{i}}\left(1-\Phi_{c}^{2}\right)} \\
\eta_{t}=\eta_{t}\left(\Phi_{c}, \omega, T_{i}\right)
\end{gathered}
$$

Where $K$ is a parameter. These explicit expressions of $\dot{m}$ and $\eta_{t}$ avoid the algebraic loops previously referred.

\section{Simulation Results}

The composition diagram of the compressor-turbine system Modelica model is shown in Fig. 7. For the compressor model, the above explained first principles approximation for the compressor map from (12) to (17) has been implemented. For the turbine module, the Stodola model has been applied based on (18) and (19).

The experiment which results are shown in Fig. 8 consists of applying a sequence of ramps for input heat power to the combustor, equivalent to gas mass flow rate ramps. A step for external torque is applied from the beginning, emulating a cold start-up to reach initial conditions inside the validity region of the model. These ramps are represented in u_ThermalPowerPercent and u_TorquePercent as variables representing the percentage of the total thermal power injected and the torque applied to the axis. The variables returned from the simulation are: $f$ the frequency of the axis, mdot_compressor the mass flow rate of air through the compressor and T_Combustor the temperature inside combustor. It can be observed a (non-linear) first order behavior for the three simulated variables when assuming the input ramps as quasi-steps, due to the short up/down times. Different time constants and steady state gains must be found for each operation point and step applied. As shown, T_Combustor rises at the time of torque negative edge due to the decreasing change in mass

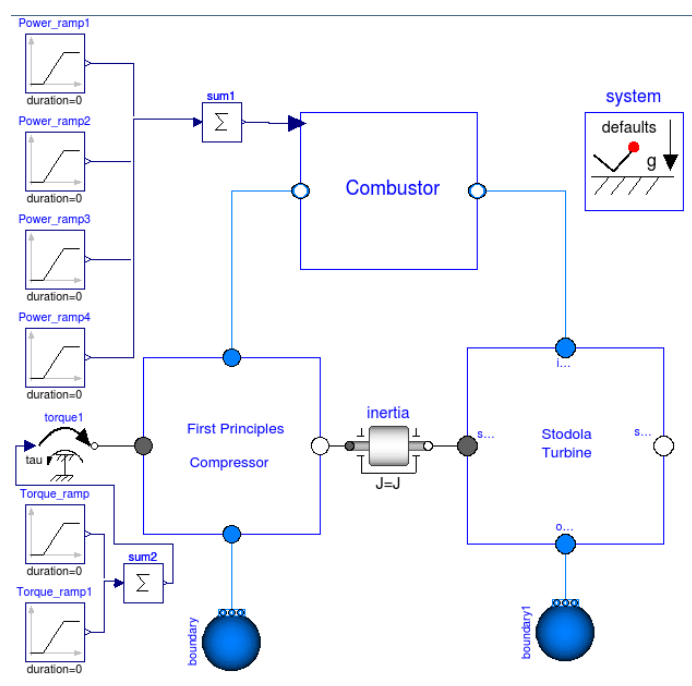

Figure 7. Diagram of the compressor-turbine system in Dymola tool. First principles compressor map and Stodola turbine models have been used. For both inputs (heat flow into combustor and torque for cold start-up) a combination of ramps are used.

flow rate, that increases residence time of fluid in the combustion chamber. This figure shows the exhaust flue gases temperature T_Out_Turbine, which is different from the temperature in the combustor.

From a control point of view, due to the restrictions of the model validity region imposed by the presented equations, only input thermal power may be used as manipulated variable and the torque may be used as disturbance.

\section{Conclusions}

A gas turbine dynamic model composed of different components has been presented. The main objective of the model is to be used in real time simulations and control applications. Only the main dynamics of a gas turbine need to be predicted in a wide operation range and with the minimum complexity to obtain a dynamic inverted model to be used inside the controllers. An analysis is presented on computational causality in the initialization and the IVP with concluding requirements for numerical convergence. A proposal for compression and expansion maps, derived from first principles are presented, to be used when unavailability of data from manufacturer and avoiding the formation of algebraic loops. Some simulation experiments are performed and the results shown.

\section{Acknowledgment}

The authors gratefully acknowledge the funding support from CIEMAT Research Centre, EU $7^{\text {th }}$ Framework Programme (Theme Energy 2012.2.5.2) under grant agreement 308912 - HYSOL project - Innovative Configuration of a Fully Renewable Hybrid CSP Plant, the National R+D+i Plan Project DPI2014-56364-C2-2-R of the Spanish Ministry of Economy and Competitiveness and ERDF funds. 

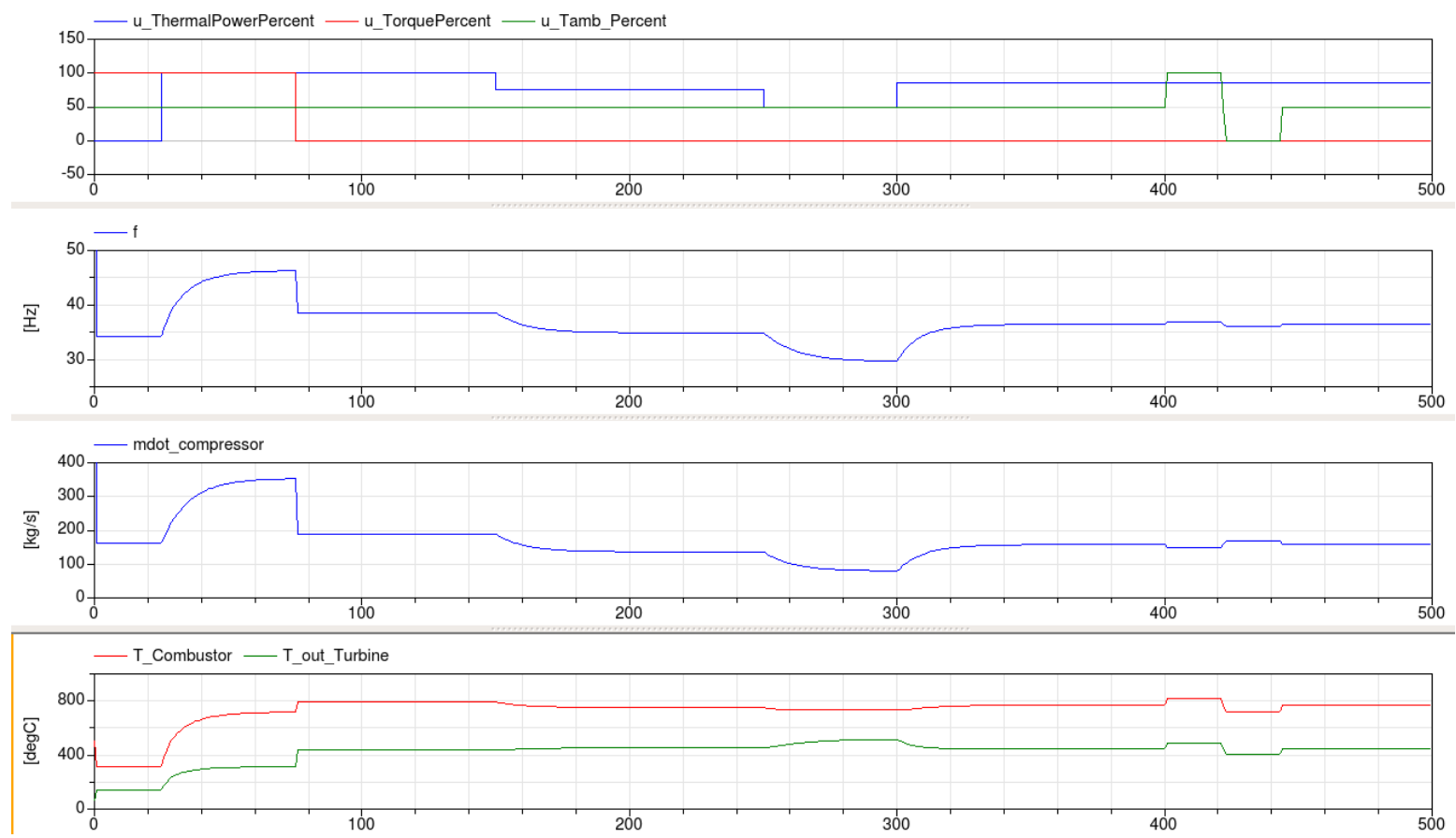

Figure 8. Input variable and simulation results for the model in Fig. 7.

\section{References}

3DS. Dymola 2016 User Manual, 2016.

W. W. Bathie. Fundamentals of gas turbines. Wiley, second edition, 1996. ISBN 9780471311225.

F. Casella and A. Leva. Modelling of thermo-hydraulic power generation processes using Modelica. Mathematical and Computer Modelling of Dynamical Systems, 12(1):19-33, 2006. doi:10.1080/13873950500071082.

F. Casella, M. Otter, K. Proelss, C. Richter, and H. Tummescheit. The modelica fluid and media library for modeling of incompressible and compressible thermo-fluid pipe networks. In Proceedings of the Modelica Conference, pages 631-640, 2006.

F. E. Cellier. Continuous System Modeling. Springer-Verlag, 1991.

H. Elmqvist, H. Tummescheit, and M. Otter. Object-oriented modeling of thermo-fluid systems. In 3rd International Modelica Conference, pages 269-286, 2003.

P. Fritzson. Principles of object-oriented modeling and simulation with Modelica 2.1. Wiley-IEEE Press, 2004.

J. T. Gravdahl and O. Egeland. Moore-greitzer axial compressor model with spool dynamics. In Proceedings of the IEEE Conference on Decision and Control, volume 5, pages 47144719. IEEE, 1997.

J. T. Gravdahl and O. Egeland. Centrifugal compressor surge and speed control. IEEE Transactions on Control Systems Technology, 7(5):567-579, 1999. ISSN 10636536. doi:10.1109/87.784420.
J. T. Gravdahl, F. Willems, B. De Jager, and O. Egeland. Modeling for surge control of centrifugal compressors: Comparison with experiment. In Proceedings of the IEEE Conference on Decision and Control, volume 2, pages 1341-1346, 2000.

J. T. Gravdahl, F. Willems, B. De Jager, and O. Egeland. Modeling of surge in free-spool centrifugal compressors: Experimental validation. Journal of Propulsion and Power, 20(5): 849-857, 2004. ISSN 07484658.

E. M. Greitzer. Surge and rotating stall in axial flow compressors. part i: Theoretical compression system model. Journal of Engineering for Gas Turbines and Power, 98(2):190-198, 1976.

S. C. Gülen and K. Kim. Gas turbine combined cycle dynamic simulation: A physics based simple approach. Journal of Engineering for Gas Turbines and Power, 136(1), 2014.

R. Kehlhofer, B. Rukes, F. Hannemann, and F. Stirnimann. Combined-cycle gas \& steam turbine power plants. Pennwell Books, 2009.

S. V. Patankar. Numerical Heat Transfer and Fluid Flow. Series in Computational and Physical Processes in Mechanics and Thermal Sciences. Taylor \& Francis, Mortimer House, 37-41 Mortimer Street, London, W1T 3JH, 1980.

K. J. Åström, H. Elmqvist, and S. E. Mattsson. Evolution of Continuous-Time Modeling and Simulation. In R Zobel and D Moeller, editors, Proceedings of the 12th European Simulation Multiconference, ESM'98, pages 9-18, Manchester, UK, June 1998. Society for Computer Simulation International.

H. Tummescheit. Design and Implementation of ObjectOriented Model Libraries using Modelica. 2002. 
H. K. Versteeg and W. Malalasekera. An Introduction to Computational Fluid Dynamics. Addison Wesley Longman Limited, Pearson Education. Edinburgh Gate. Harlow. CM20 2JE. United Kingdom., 1995.

\section{Appendix}

From (Gravdahl and Egeland, 1999) and its references, the remaining equations and parameters for the model are exposed below. All the parameters are in table 1. Slip factor $\sigma$ in (12) is a parameter that depends on the construction but is assumed to be minor but close to 1 . The expression used is (20). Parameter $\alpha$ is defined by (21).

$$
\begin{gathered}
\sigma=1-2 / N_{b} \\
\alpha=\frac{A \cot \left(\beta_{1 b}\right)}{A_{i} r_{1} \rho_{i}}
\end{gathered}
$$

The total friction factor $k_{f}$ in (22) is the overall friction factor from all friction losses.

$$
k_{f}=\frac{4 f l}{2 D \rho_{i}^{2} A_{i}^{2} \sin ^{2}\left(\beta_{1 b}\right)}
$$

For friction factor expression any of the bibliography can be used, although the Blasius' formula is one of the most frequently used (23). In this case, $f$ has been considered a parameter for the sake of simplicity.

$$
f=0.3164 R e^{-0.25}
$$

The dependent parameters $\left\{a_{01}, a_{2}\right\}$ are defined by (24) and (25).

$$
\begin{aligned}
& a_{01}=\frac{r_{1}^{2}}{2}-\sigma r_{2}^{2} \\
& a_{2}=\frac{\alpha^{2} r_{1}^{2}}{2}+k_{f}
\end{aligned}
$$

Table 1. Models Parameters

\begin{tabular}{cc}
\hline Parameter & Magnitude \\
\hline$N_{b}$ & Number of compressor blades \\
$f$ & Friction factor \\
$l$ & Mean channel length in the compressor \\
$D$ & Mean hydraulic diameter \\
& for the compressor \\
$A$ & Section of the circle with diameter $D$ \\
$A_{i}$ & Inlet section of the compressor \\
$\rho_{i}$ & Inlet air density \\
$\beta_{1 b}$ & Backswept inlet vane angle \\
$r_{2}$ & Impeller outer radius \\
$r_{1}$ & Impeller inner radius \\
\hline
\end{tabular}

